\title{
Enamel bevelling and retention of composite restorations in non-carious cervical lesions
}

\author{
Abstracted from \\ Schroeder M, Reis A, Luque-Martinez I, Loguercio AD, Masterson D, Maia LC. \\ Effect of enamel bevel on retention of cervical composite resin restorations: \\ A systematic review and meta-analysis. / Dent 2015; 43: 777-788. \\ Address for correspondence: Lucianne Cople Maia, Federal University of Rio de Janeiro, \\ Department of Pediatric Dentistry and Orthodontic, Postal code: 68066, Cidade Universitaria, \\ CEP 21941-971, Rio de Janeiro, RJ, Brazil. E-mail: rorefa@terra.com.br
}

\section{Question: Does enamel bevelling affect the retention of composite restorations in non- carious cervical lesions?}

Data sources Medline, Web of Science, Latin American and Caribbean Health Sciences Literature database (LILACS), Brazilian Library in Dentistry (BBO), the Cochrane Library, abstracts of the annual conference of the International Association for Dental Research (IADR), System for Information on Grey literature in Europe (SIGLE), ProQuest Dissertations and Theses Fulltext database as well as the Periódicos Capes Theses, Current Controlled Trials, International Clinical trials registry platform, the ClinicalTrials.gov, Rebec and the EU Clinical Trials Register.

Study selection Randomised clinical trials (RCTs) that compared the retention rates of restorations in NCCLs placed with or without bevel with at least one year follow-up were considered.

Data extraction and synthesis Data were abstracted using standardised forms and study quality was assessed using the Cochrane risk of bias tool. Marginal discolouration scores were dichotomised into yes and no, and risk differences for retention rate and marginal discolouration were calculated for each study for analysis.

Results Four studies were included. Two studies were considered to be at high risk of bias and not included in the meta-analysis. The overall risk difference was $0.0(95 \% \mathrm{Cl} ; 0.04$ to 0.04$)$ for the retention rate and $0.05((95 \% \mathrm{Cl} ; 0.02$ to 0.13$)$ for the marginal discolouration, suggesting that enamel beveling does not influence retention rate or marginal discolouration.

Conclusions One may conclude that there is no difference between bevelled and non-bevelled technique over the short-term follow-up of 12-18 months of clinical service, although this conclusion was based on only two low risk of bias RCTs. Additionally, there is not enough evidence to support this conclusion over longer-term followups. There is a need for better standardisation and the reporting of RCTs investigating this technique variation after longer-term followup periods.

\section{Commentary}

Non Carious Cervical Lesions (NCCLs) have been the subject of much debate, especially in reference to their aetiology ${ }^{1}$ and management. ${ }^{2}$ Moreover, the reported prevalence of NCCLs varies widely, from $2 \%-90 \%$ of the population, depending on the type of study, methodology, and participants. ${ }^{3}$ As such, the importance of having clear clinical recommendations, based on robust evidence, becomes pertinent. One of the methods of evaluating the overall quality of the available body of evidence is performing a systematic review, with/out meta-analyses. The current systematic review ${ }^{4}$ investigated the effect of enamel bevelling on the retention and marginal discolouration of composite restorations of NCCLs. The methodology employed in the review was rigorous, with a registered study protocol, multiple database search, no restrictions on language or date of publication, a risk of bias analysis, and largely complying with the Preferred Reporting Items for Systematic Reviews and MetaAnalyses guidelines (PRISMA). ${ }^{5}$

The review concluded that there was a lack of evidence supporting bevelling enamel when restoring NCCLs. The authors, however, acknowledge that the review had a number of limitations regarding the included studies and follow-up duration. The systematic review only included four randomised clinical trials, with two trials later excluded from the meta-analysis due to high risk of bias. Consequently, the meta-analysis findings were based only on two studies, involving the placement of 204 composite restorations in 76 patients. The retention rates of the composite restorations ranged between $88 \%$ and $100 \%$ over a short follow-up period of 1-1.5 years, and a drop out rate of $27 \%$. Details of restoration technique/ protocol employed varied widely between included studies, with the use of different restorative materials and burs, and differences in the length and angle of prepared bevel. Furthermore, one study placed the restorations under rubber dam isolation while the other did not.

The findings of the current review seem to confirm those of an earlier systematic review ${ }^{6}$ investigating the retention and performance of cervical restorations using various adhesive systems, restorative materials and restoration protocols. In contrast to the current review, the Heintz et al. review was not limited to randomised clinical trials, and as such included fifty prospective clinical trials with a minimum duration of 1.5 years, and with no risk of bias analysis performed. The review concluded that, indeed, bevelling of enamel and the use of rubber dam isolation had no significant influence on the performance 
of the restorations. The use of 2-step self-etching or 3-step etch and rinse adhesive systems, glass ionomer-based restorations, and preparing/roughening of the enamel and dentine did, however, have a positive influence on the performance of cervical restorations.

The strength of clinical recommendation, derived solely from the current systematic review, is limited. However, when considered in conjunction with previously existing literature, the current review further confirms that bevelling of enamel in NCCLs does not affect the performance of cervical restorations in the short-term. Evidence supporting long-term performance, in the form of standardised and randomised clinical trials, remains lacking.
Khaled Ahmed

Faculty of Dentistry, University of Hong Kong, Hong Kong

1. Silva AG, Martins CC, Zina LG, et al. The association between occlusal factors and noncarious cervical lesions: A systematic review, / Dent 2013; 41: 9-16.

2. Estafan A, Bartlett D, Goldstein G. A survey of management strategies for noncarious cervical lesions. Int J Prosthodont 2014; 27: 87-90.

3. Troendle KB, Gureckis KM. Noncarious Cervical Lesions: Prevalence, Etiology, and Management. In: Amaechi TB (ed). Dental Erosion and Its Clinical Management. Cham: Springer International Publishing; 2015: 249-273.

4. Schroeder M, Reis A, Luque-Martinez I, Loguercio AD, Masterson D, Maia LC. Effect of enamel bevel on retention of cervical composite resin restorations: A systematic review and meta-analysis, J Dent 2015; 43: 777-788.

5. Moher D, Shamseer L, Clarke M, et al. Preferred reporting items for systematic review and meta-analysis protocols (PRISMA-P) 2015 statement. Syst Rev 2015; 4: 1.

6. Heintze SD, Ruffieux C, Rousson V. Clinical performance of cervical restorations--a meta-analysis, Dent Mater 2010; 26: 993-1000.

Evidence-Based Dentistry (2016) 17, 88-89. doi:10.1038/sj.ebd.6401191 